\title{
Insurance Coverage Predicts Mortality in Patients Transferred Between Hospitals: a Cross-Sectional Study
}

\author{
Michael G. Usher, $M D P h D^{7}$, Christine Fanning, $M D^{2}$, Vivian W. Fang, $P h D^{3}$, Madeline Carroll, $M D^{2}$, \\ Amay Parikh, MD MBA MS4, Anne Joseph, $\mathrm{MD} \mathrm{MPH}^{7}$, and Dana Herrigel, $\mathrm{MD}^{5}$
}

'Department of Medicine, Division of General Internal Medicine, University of Minnesota Medical School, Minneapolis, MN, USA; ${ }^{2}$ Department of Medicine, Division of General Internal Medicine, Rutgers, Robert Wood Johnson Medical School, New Brunswick, NJ, USA; ${ }^{3}$ Department of Accounting, Carlson School of Management, University of Minnesota, Minneapolis, MN, USA; ${ }^{4}$ Department of Medicine, Divisions of Nephrology and Critical Care, Rutgers, Robert Wood Johnson Medical School, New Brunswick, NJ, USA; ${ }^{5}$ Department of Hospital Internal Medicine, Mayo Clinic Florida, Jacksonville, FL, USA.

BACKGROUND: Patients transferred between hospitals are at high risk of adverse events and mortality. The relationship between insurance status, transfer practices, and outcomes has not been definitively characterized.

OBJECTIVE: To identify the association between insurance coverage and mortality of patients transferred between hospitals.

DESIGN: We conducted a single-institution observational study, and validated results using a national administrative database of inter-hospital transfers.

SETTING: Three ICUs at an academic tertiary care center validated by a nationally representative sample of interhospital transfers.

PATIENTS: The single-institution analysis included 652 consecutive patients transferred from 57 hospitals between 2011 and 2012. The administrative database included 353,018 patients transferred between 437 hospitals.

MEASUREMENTS: Adjusted inpatient mortality and 24h mortality, stratified by insurance status.

RESULTS: Of 652 consecutive transfers to three ICUs, we observed that uninsured patients had higher adjusted inpatient mortality (OR 2.67, $p=0.021$ ) when controlling for age, race, gender, Apache-II, and whether the patient was transferred from an ED. Uninsured were more likely to be transferred from $\mathrm{ED}$ (OR 2.3, $p=0.026$ ), and earlier in their hospital course ( 3.9 vs 2.0 days, $p=0.002$ ). Using an administrative dataset, we validated these observations, finding that the uninsured had higher adjusted inpatient mortality (OR $1.24,95 \%$ CI 1.13-1.36, $p<0.001)$ and higher mortality within $24 \mathrm{~h}$ (OR 1.33 95\% CI 1.11-1.60, $p<0.002$ ). The increase in mortality was independent of patient demographics, referral patterns, or diagnoses.

LIMITATIONS: This is an observational study where transfer appropriateness cannot be directly assessed.

CONCLUSIONS: Uninsured patients are more likely to be transferred from an ED and have higher mortality. These data suggest factors that drive inter-hospital transfer of uninsured patients have the potential to exacerbate outcome disparities.

Electronic supplementary material The online version of this article (https://doi.org/10.1007/s11606-018-4687-0) contains supplementary material, which is available to authorized users.

Received April 23, 2018

Revised June 30, 2018

Accepted July 22, 2018

Published online October 1, 2018
KEY WORDS: inter-hospital transfers; health disparities; insurance; hospital ownership.

J Gen Intern Med 33(12):2078-84

DOI: $10.1007 / \mathrm{s} 11606-018-4687-0$

(C) Society of General Internal Medicine 2018

\section{INTRODUCTION}

Incomplete insurance coverage is an important contributor to health disparities in the USA. Patients who lack insurance have decreased access to ambulatory care and chronic disease management. ${ }^{1-3}$ Uninsured critically ill patients receive less intense therapy and fewer procedures, and are more likely to have life-sustaining care withdrawn. ${ }^{4-6}$ Adjusted mortality and cause-specific mortality rates are higher among uninsured patients than those among patients with insurance., 7-9

Despite higher mortality and lack of preventive services, US health care policy has not been successful in systematizing care of the uninsured. The Burton-Hill Act of 1946 mandated uncompensated care from hospitals receiving government funding, but was nearly unenforced for 30 years. ${ }^{10}$ The Emergency Medical Treatment and Labor Act (EMTALA), passed in 1986, mandated emergency services for patients who lack the ability to pay, but provided no funding mechanism for their care. ${ }^{11}$ Finally, the Affordable Care Act, passed in 2010, made significant reductions in the numbers of uninsured; however, legal challenges have prevented universal adoption. ${ }^{12}$

Since care of the uninsured often remains uncompensated, delivery may be skewed by economic considerations leading to suboptimal care or transfer to another facility. ${ }^{13}$ Hospital to hospital transfers represent a mechanism to adjust a hospital's case-mix and payer-mix ${ }^{14}$; hospital ownership and finances may play an important and underappreciated role in hospital admission and transfer patterns, particularly in the uninsured. ${ }^{15}$ In addition to providing an opportunity to assess how medical decision making is affected by a patient's insurance status, inter-hospital transfers are an important and understudied population. Patients transferred from other acute care facilities make up $8 \%$ of critical care admissions, and 
represent approximately 1.6 million admissions annually and cost in excess of $\$ 100$ billion. $^{16,17}$

While EMTALA is designed to protect uninsured patients from unsafe transfer, it neither mitigates the primary motivation nor the risks associated with an inter-hospital transfer. We demonstrated previously that EMTALA is not effective at ensuring the transfer of complete documentation with patients who are transferred between hospitals, and that the loss of information is associated with higher risk of mortality. ${ }^{18} \mathrm{Ad}-$ ditional studies have shown that patients transferred between hospitals have elevated risk-adjusted mortality compared to non-transferred patients. ${ }^{19}$ It is a reasonable hypothesis that, despite EMTALA, unnecessary transfers of uninsured patients still occur and those patients are at elevated risk of morbidity and mortality.

One might expect that a disproportionate number of uninsured patients are transferred to other hospitals in an effort to deflect cost, particularly in an environment of increased hospital privatization. Results from studies of the rates of transfer of uninsured patients are mixed. Analysis of the Health Care Utilization Project (HCUP) National Inpatient Sample revealed that uninsured patients are significantly underrepresented among those transferred between facilities, suggesting lack of insurance is more of a barrier to transfer than motivation. ${ }^{20}$ These findings are not consistent with observations that uninsured patients presenting to the emergency department are more likely to be transferred than their insured counterpart. ${ }^{21-23}$ The subsequent outcomes of transferred patients regardless of their insurance status remain understudied.

To address this important knowledge gap, we investigated the association of insurance coverage with inter-hospital transfers and their subsequent outcomes. First, we performed a retrospective observational study of consecutive transfers to a tertiary referral center. To validate our findings, we repeated the analysis using a nationally representative database of interhospital transfers where both the referring and receiving hospital characteristics could be described.

\section{MATERIALS AND METHODS}

The study approach included an exploratory analysis of consecutive patient transfers to a tertiary referral center focusing on how insurance status, transfer practices, and subsequent mortality were related. Critical care transfers were selected as the population of interest to enrich event rates for this individual chart review study. We then validated our findings in an administrative data set of hospital transfers. IRB approval was obtained from both Rutgers University and the University of Minnesota.

\section{Data Sources}

Single-Institution Observational Study. We conducted a retrospective observational study of patients transferred to the three ICUs (Medical ICU, Surgical ICU, and Cardiac
Care Unit) of the Robert Wood Johnson University Hospital (RWJUH). RWJUH is a 610-bed tertiary academic medical center located in New Brunswick, NJ, and is the principal teaching hospital of Rutgers' Robert Wood Johnson Medical School. It is a not-for-profit, privately owned hospital which receives considerable governmental support from New Jersey Charity Care. Consecutive transfers between January 1, 2011, and December 31, 2012, were identified through transfer center records. Inclusion criteria encompassed all patients aged 18 years or older, directly transferred to a RWJUH ICU from outside hospital critical care units or emergency departments (ED). Only the first ICU admission for each inpatient encounter was included for analysis to limit the bias caused by repeat ICU readmissions.

Administrative Database Study. To support the generalizability of these findings and confirm they were not limited to critical care transfers or isolated to a single tertiary care center, we developed a large administrative database. Patients were identified from a cohort of hospital to hospital transfers from the Heath Care Utilization Project (HCUP) State Inpatient Databases (SID) and State Emergency Department Database (SEDD) from NY, FL, UT, IA, and VT from 2011 through $2013 .{ }^{24}$ These states were used as they contain a unique "visitlink" patient identifier that allows patient tracking across ED and inpatient visits as well as ability to merge with the American Hospital Association annual survey. Interhospital transfers were first identified by linking individual patients with identical age, race, gender, and unique visitlink ID by discharge and admission date and designation of a transfer to or from an acute care facility.

\section{Outcomes and Measures}

Single-Institution Observational Study. Patient primary payer information was extracted from hospital records. If insurance status was unknown, they were excluded from the study. The primary exposure variable was insurance status of the patient. We did not compare individual insurance types due to a lack of statistical power. Severity of illness was calculated using Apache-II. ${ }^{25}$ Measures of resource utilization included ICU length of stay and total length of stay. Additional measures included prior hospital length of stay (defined by the date of initial admission to the moment of transfer) and ED versus inpatient origin. The primary outcome was inpatient mortality.

Administrative Database Study. The primary exposure variable was insurance status, which was grouped into private, Medicare, Medicaid, or uninsured as indicated at the receiving hospital. Patients without a known insurance were excluded. We compared components of the originating hospital stay including length of stay, emergency department (ED) or inpatient origin, and hospital ownership as defined by the American Hospital Association annual survey stratified into government (non-federal), non-profit, or for-profit investor owned. We additionally collected age, race, gender, 
Elixhauser comorbidities, admitting diagnosis at the receiving hospital, and median income quartile of the patient's home zip code. The primary outcome was inpatient mortality, with secondary measures including mortality within $24 \mathrm{~h}$ of transfer as a measure of instability and discharge within $48 \mathrm{~h}$ as a measure of unnecessary transfer.

Finally, we investigated the rate of transfer to a higher level of care for transfers involving stroke (CVA) or acute myocardial infarction (AMI). For CVA, this was defined by transfers from a hospital without fibrinolysis capability to a hospital with sufficient fibrinolysis cases to be included in hospital quality reporting (measure SK4, 2015 reporting). Similarly, for acute myocardial infarction, we collected the rate patients were transferred from hospitals without PCI capability to hospitals with PCI quality reporting (AMI-8a, 2015 reporting).

\section{Analysis}

Single-Institution Observational Study. Demographic information was included as a number and percentage if a dichotomous variable and a mean and standard deviation for continuous variable. We compared components of the prior hospital stay by chi-square and $t$ test as appropriate with $p<0.05$ to denote statistical significance. For length of stay, we compared log-transformed values. For adjusted inpatient mortality, multivariate logistic regression was used to evaluate the effect of insurance coverage on mortality controlling for age, gender, race, emergency department origin, and ApacheII as a covariate.

Administrative Database Study. We took a parallel approach in the large administrative dataset analysis. Chi-square test was used to determine whether dichotomous variables varied by insurance status. One-way ANOVA test was used to determine differences between insurance groups for continuous variables, using $\log$ transformation as indicated. Betweengroup comparisons were made using private insurance as a reference, adjusting for repeated measures using Bonferroni. Defining privately insured patients as the reference group, we utilized multilevel logistic regression to adjust for clustering by the receiving hospital.

Since uninsured patients differed by diagnoses, demographics, and hospital utilization, we took to approaches to stratification. First, we investigated mortality in 24-h and allcause inpatient mortality while stratifying by age, race, whether patient was transferred from emergency department, hospital ownership, and Elixhauser comorbidities. Since traumas made up a disproportionate admitting diagnosis of the uninsured, we performed a sensitivity analysis excluding admissions with physical injury as the admitting diagnosis of the receiving hospital (Clinical Classification Score, CCS 225236, 239-244). We additionally performed several subpopulation analyses. We investigated risk-adjusted inpatient mortality across the three most common medical diagnoses across insurance coverage: Acute myocardial infarction (CCS 100, 101), acute cerebrovascular disease (CCS 109). We performed analysis across age, race, gender, hospital ownership structure, and whether they were transferred from the emergency department (holding one variable constant, while adjusting for all others). We present overall odds ratio and 95\% confidence intervals of mortality stratified by hospital ownership and insurance coverage. STATA v13 (StataCorp; College Station, TX) was used for all statistical analyses.

\section{RESULTS}

\section{Insurance Coverage and Patient Transfers}

Single-Institution Observational Study. Between January 2011 and December 2012, 652 patients were transferred directly to RWJ ICUs from 57 different hospitals. Patient demographics and outcomes are summarized in Table 1. Patients without insurance had a shorter ICU $(p=0.025)$ and hospital length of stay $(p<0.001)$, compared to the insured cohort. Unadjusted mortality was similar between the two groups. Patients without insurance were transferred earlier in the original hospitalization (2.02 days vs 3.86 days, $p=$ 0.002 ), more likely to be transferred directly from the emergency department $(p=0.016)$, and more likely to arrive at night ( 65 vs $49.7 \%, p=0.046$ ).

Administrative Database Study. To demonstrate our findings were not biased by practices of a single hospital system nor by the relatively small numbers of uninsured patients, we queried a large administrative database of patients transferred between

Table 1 Patient Demographics and Outcomes of a Pilot Observational Study of Consecutive Transfers to Three ICUs: Single-Institution Pilot Study $(N=652)$

\begin{tabular}{|c|c|c|c|c|}
\hline & & Insured & Uninsured & $p$ \\
\hline \multicolumn{2}{|l|}{$N$} & 599 & 53 & N/A \\
\hline \multicolumn{2}{|l|}{ Age (SD) } & $54.6(12.3)$ & $47.3(9.4)$ & $<0.001$ \\
\hline \multicolumn{2}{|l|}{ Male (\%) } & $351(58.6)$ & $41(77.4)$ & $<0.001$ \\
\hline \multirow[t]{3}{*}{ Race } & White & $442(73.8)$ & $26(49.1)$ & $<0.001$ \\
\hline & Black & $56(9.3)$ & $5(9.4)$ & \\
\hline & Other & $101(16.8)$ & $22(41.5)$ & \\
\hline \multirow[t]{3}{*}{ Initial ICU } & MICU & 107 (17.9) & $11(20.7)$ & 0.058 \\
\hline & SICU & $250(41.7)$ & $29(54.7)$ & \\
\hline & $\mathrm{CCU}$ & $242(40.4)$ & $13(24.5)$ & \\
\hline \multicolumn{2}{|c|}{$\begin{array}{l}\text { Prior hospitalization } \\
\text { days (SD) }\end{array}$} & $3.86(7.1)$ & $2.02(3.6)$ & 0.002 \\
\hline \multicolumn{2}{|c|}{ Transferred from } & $203(33.9)$ & $27(50.9)$ & 0.016 \\
\hline \multicolumn{2}{|c|}{$\begin{array}{l}\text { Arrived on } \\
\text { weekend }(\%)\end{array}$} & $154(25.7)$ & $19(35.8)$ & 0.142 \\
\hline \multicolumn{2}{|c|}{ Arrived at night (\%) } & $298(49.7)$ & $34(64.1)$ & 0.046 \\
\hline \multicolumn{2}{|c|}{$\begin{array}{l}\text { ICU LOS days (SD) } \\
\text { Hospital LOS days }\end{array}$} & $4.6(5.4)$ & $2.8(3.2)$ & 0.025 \\
\hline \multicolumn{2}{|c|}{$\begin{array}{l}\text { Hospital LOS days } \\
\text { (SD) }\end{array}$} & $11.83(15.7)$ & $6.74(5.7)$ & $<0.001$ \\
\hline \multicolumn{2}{|c|}{ Apache-II (SD) } & $14.96(9.0)$ & $10.4(9.5)$ & 0.022 \\
\hline \multicolumn{2}{|c|}{ MPM0-III (SD) } & $0.23(0.21)$ & $0.11(0.2)$ & $<0.00$ \\
\hline \multicolumn{2}{|c|}{$\begin{array}{l}\text { Inhospital } \\
\text { mortality }(\%)\end{array}$} & $105(17.7)$ & $10(18.8)$ & 1 \\
\hline \multicolumn{2}{|c|}{$\begin{array}{l}\text { Adjusted inpatient } \\
\text { mortality* }\end{array}$} & 1 (reference) & $2.67(1.16-6.16)$ & 0.021 \\
\hline
\end{tabular}

*Adjusted with multivariate logistic regression including age, gender, race, ED transfer, nighttime arrival, and Apache-II score 
hospitals. From an initial dataset of over nine million inpatient encounters, we identified 343,018 records where both hospital stays and the transfer could be confirmed. We find that patients who are transferred are younger $(p<0.001)$, less likely to be female ( 59.1 to $47.8 \%, p<0.001$ ), less racially diverse, and more likely to be uninsured ( 6.7 vs $4.4 \%, p<0.001)$.

Patient demographics and outcomes of patients transferred between hospitals, stratified by insurance coverage, are summarized in Table 2. On average, there was a net movement of patients from/to government-owned facilities following transfer ( $16.1 \%$ prior to transfer, $18.2 \%$ following transfer). Patients with Medicaid (22.2\%) and the uninsured (23.4\%) were more likely to be transferred to a government-owned hospital. Compared to patients with private insurance, patients who were uninsured were transferred earlier (1.2 vs 2.7 days, $p<0.001)$ and more likely to be transferred from emergency departments $(75.9$ vs $59.3 \%, p<0.001)$.

Across all insurance coverages, the most common admitting diagnosis was acute myocardial infarction making up $10.4 \%$ of privately insured patient transfers, $10.9 \%$ of Medicare transfers, $4.9 \%$ of Medicaid transfers, and $10.5 \%$ of uninsured transfers. Traumas, including intracranial injury, skull and face fractures, and crushing injury, were more common among the uninsured than patients with private insurance (15.1 vs $11.1 \%$ ) (Supplemental Table II).

\section{Mortality of Inter-Hospital Transfers}

Single-Institution Observational Study. Rates of unadjusted mortality between insured (17.7\%) and uninsured transfers (18.8\%) were similar. Illness severity measured by both Apache-II and MPM0-III was significantly lower among un- insured patients. Multivariate analysis, controlling for age, race, ED origin, and Apache II score, of uninsured patients exhibited a higher adjusted inpatient mortality (OR 2.67, 95\% CI 1.16-6.16, $p=0.021)$.

Administrative Database Study. All-cause adjusted inpatient mortality, controlling for age, race, Elixhauser comorbidities, whether the patient was transferred directly from another emergency department, and adjusting for clustering by hospital, demonstrated that uninsured patients had higher mortality when compared to patients with private insurance (OR 1.24, 95\% CI 1.13-1.36, $p<0.001$, Table 3). Adjusted, 24-h mortality was also significantly higher (OR 1.33, 95\% CI 1.11-1.60, $p=0.002$ ). We performed a sensitivity analysis excluding patient admissions with trauma diagnoses. This was because traumas would be less sensitive to preventive services and care coordination afforded by health insurance. We found adjusted 24-h mortality and inpatient mortality remained higher among the uninsured. Finally, we investigated cause-specific mortality for the most common medical reasons for transfer, finding that uninsured patients had higher inpatient mortality when transferred with AMI (OR $1.4395 \%$ CI $1.06-1.95, p=0.025)$ and CVA (OR 1.52, 95\% CI 1.24-1.87, $p<0.001$ ), and not significantly higher with sepsis (OR 1.15, 95\% CI 0.85-1.55). Uninsured patients required transfer to a higher level of care for PCI services $78.2 \%$ of the time which was similar to patients with private insurance (77.6\%). Uninsured patients with CVA were transferred to a higher level of care at a slightly higher rate as patients with private insurance (48.1 vs $50.0 \%$, Supplemental Table III).

Table 2 Summary of Insurance Coverage on Transfer Patterns and Outcomes: National Administrative Database of Inter-Hospital Transfers $(n=343,018)$

\begin{tabular}{|c|c|c|c|c|c|}
\hline & Private & Medicare & Medicaid & Uninsured & $p$ \\
\hline$N$ & 85,692 & 174,715 & 61,547 & 21,064 & \\
\hline Age (SD) & $46.4(20.4)$ & $72.9(12.3)^{*}$ & $30.1(22.1)^{*}$ & $42.7(16.8)^{*}$ & $<0.001$ \\
\hline Female (\%) & $37,908(44.2 \%)$ & $86,814(48.6 \%)$ & $41,724(51.5 \%)$ & $7521(35.7 \%)$ & $<0.001$ \\
\hline White & $63,820(74.5 \%)$ & $133,116(74.5 \%)$ & $29,729(48.3 \%)$ & $15,333(63.6 \%)$ & $<0.001$ \\
\hline Black & $7942(9.3)$ & $17,064(9.6 \%)$ & $13,813(22.4 \%)$ & $3942(16.3 \%)$ & \\
\hline Hispanic & $7098(8.3 \%)$ & $18,640(10.4 \%)$ & $11,914(19.4 \%)$ & $2962(12.3 \%)$ & \\
\hline Other & $6834(8.0 \%)$ & $8745(5.5 \%)$ & $6091(9.9 \%)$ & $1876(7.8 \%)$ & \\
\hline \multicolumn{6}{|l|}{ Referring hospitalization } \\
\hline Referring hospital LOS (SD) & $2.78(8.0)$ & $4.45(8.7)^{\dagger}$ & $2.5(9.7)^{\dagger}$ & $1.2(5.0)^{\dagger}$ & $<0.001$ \\
\hline Transferred from referring ED (\%) & $50,824(59.3 \%)$ & $74,960(42.0 \%)$ & $40,237(65.4 \%)$ & $18,312(75.9 \%)$ & $<0.001$ \\
\hline Total charges & $31,674(94 \mathrm{k})$ & $48,172(103 \mathrm{k})^{\dagger}$ & $21,869(72 \mathrm{k})^{\dagger}$ & $15,556(38 \mathrm{k})^{\dagger}$ & $<0.001$ \\
\hline Investor owned (for profit) & $15,432(18.0 \%)$ & $46,840(26.2 \%)$ & $15,102(24.5 \%)$ & $7129(30.0 \%)$ & $<0.001$ \\
\hline Non-profit owned & $59,999(70.0 \%)$ & $108,771(60.9 \%)$ & $35,660(57.9 \%)$ & $12,635(52.3 \%)$ & \\
\hline Government owned & $9406(11.0 \%)$ & $31,448(12.0 \%)$ & $10,378(16.8 \%)$ & $4187(17.4)$ & \\
\hline \multicolumn{6}{|l|}{ Receiving hospitalization } \\
\hline LOS (days, SD) & $7.1(11.3)$ & $10.1(13.0)^{\dagger}$ & $6.6(11.7)^{\dagger}$ & $5.5(9.2)^{\dagger}$ & $<0.001$ \\
\hline Total charges $(\$, S D)$ & $71,618(118 \mathrm{k})$ & $83,475(112 \mathrm{k})^{\dagger}$ & $60,568(117 \mathrm{k})^{\dagger}$ & $64,352(107 \mathrm{k})^{\dagger}$ & $<0.001$ \\
\hline Investor owned (for profit) & $11,222(13.1 \%)$ & $46,318(25.9 \%)$ & $7815(12.7 \%)$ & $4613(19.1 \%)$ & $<0.001$ \\
\hline Non-profit owned & $57,193(66.7 \%)$ & $105,689(59.2 \%)$ & $39,834(65.7 \%)$ & $13,823(57.3 \%)$ & \\
\hline Government owned & $17,062(20.0 \%)$ & $25,984(14.5 \%)$ & $13,637(22.2 \%)$ & $5639(23.4 \%)$ & \\
\hline Elixhauser comorbidity sum (IQR) & $2.91(2.57)$ & $5.18(2.74)^{\dagger}$ & $2.43(2.49)$ & $2.3(2.2)^{\dagger}$ & $<0.001$ \\
\hline Discharged within $48 \mathrm{~h}$ of transfer & $25,975(30.3 \%)$ & $33,329(18.7 \%)$ & $20,245(32.9 \%)$ & $7740(32.1 \%)$ & $<0.001$ \\
\hline Mortality within $24 \mathrm{~h}$ of transfer & $522(0.6 \%)$ & $2088(1.2 \%)$ & $262(0.4 \%)$ & $158(0.7 \%)$ & $<0.001$ \\
\hline Inpatient mortality & $3487(4.3 \%)$ & $16,621(9.5 \%)$ & $1982(3.5 \%)$ & $648(3.1 \%)$ & $<0.001$ \\
\hline
\end{tabular}

$*_{p}<0.002$ by one-way ANOVA, compared with privately insured

$p<0.002$ by one-way ANOVA following log transformation (pairwise comparison with privately insured) 
Table 3 Association Between Insurance Coverage and Inpatient Mortality, Stratified by Diagnosis

\begin{tabular}{|c|c|c|c|c|c|c|c|}
\hline & \multirow[t]{2}{*}{ Private } & \multicolumn{2}{|l|}{ Medicare } & \multicolumn{2}{|l|}{ Medicaid } & \multicolumn{2}{|l|}{ Uninsured } \\
\hline & & OR $(95 \% \text { CI })^{*}$ & $p$ & OR $(95 \% \mathrm{CI})^{*}$ & $p$ & OR $(95 \% \text { CI })^{*}$ & $p$ \\
\hline Overall 24-h mortality & 1.0 (reference) & $1.17(1.05-1.31)$ & 0.004 & $0.93(0.8-1.09)$ & 0.417 & $1.33(1.11-1.60)$ & 0.002 \\
\hline 24-h mortality Excluding trauma & 1.0 (reference) & $1.19(1.06-1.34)$ & 0.003 & $0.95(0.81-1.12)$ & 0.524 & $1.40(1.15-1.70)$ & 0.001 \\
\hline Overall Inpatient mortality & 1.0 (reference) & $1.07(1.02-1.12)$ & 0.004 & $1.10(1.03-1.18)$ & 0.003 & $1.24(1.13-1.36)$ & $<0.001$ \\
\hline Inpatient Mortality Excluding trauma & 1.0 (reference) & $1.08(1.04-1.14)$ & 0.001 & $1.11(1.03-1.19)$ & 0.003 & $1.29(1.17-1.42)$ & $<0.001$ \\
\hline $\mathrm{AMI}+$ & 1.0 (reference) & $1.09(0.92-1.31)$ & 0.319 & $0.98(0.73-1.32)$ & 0.919 & $1.42(1.05-1.95)$ & 0.025 \\
\hline $\mathrm{CVA}^{\dagger}$ & 1.0 (reference) & $1.29(1.11-1.50)$ & 0.001 & $1.45(1.19-1.75)$ & $<0.001$ & $1.52(1.23-1.86)$ & $<0.001$ \\
\hline Sepsis $†$ & 1.0 (reference) & $1.02(0.89-1.18)$ & 0.41 & $1.03(0.85-1.25)$ & 0.738 & $1.15(0.85-1.55)$ & 0.36 \\
\hline Intracranial injury $\dagger$ & 1.0 (reference) & $0.89(0.69-1.15)$ & 0.375 & $1.21(0.84-1.76)$ & 0.297 & $0.88(0.60-1.29)$ & 0.516 \\
\hline
\end{tabular}

*OR and $p$ value by multi-level logistic regression adjusting for age, gender, race, hospital ownership, whether the patient was transferred from an emergency department, and Elixhauser comorbidities

${ }^{\dagger}$ Admitting diagnosis of the receiving hospital, obtained via AHRQ clinical classification system

We additionally stratified by patient and hospital factors (Table 4). Uninsured patients had higher adjusted inpatient mortality across age, race, gender, and socioeconomic status as proxied by median income of a patient's zip code. Higher mortality of uninsured patients was limited to those directly transferred from emergency departments. Higher mortality of uninsured patients was limited to patients who were transferred to non-profit and government-owned hospitals, and not those transferred to for-profit investor-owned hospitals.

\section{DISCUSSION}

Incomplete medical insurance coverage is a driving force of health care disparities in the USA. We investigated the complex relationship between insurance coverage, hospital transfer practices, and patient outcomes. We demonstrate both in a single tertiary referral center and in a representative administrative database that patients without insurance are transferred earlier, often prior to admission, and have higher mortality. We found higher mortality within the first $24 \mathrm{~h}$ of transfer even when patients were transferred from emergency departments. Our findings did not appear to be explained by differences in demographics and diagnoses. Additionally, we found that rates of uninsured were slightly higher among patients who were transferred when compared to the entire inpatient sample, and that uninsured patients were more likely to be transferred to government-owned hospitals.

Despite multiple medical advancements, electronic health records adoption, and the passage of EMTALA, our findings

Table 4 Association Between Insurance Coverage and Inpatient Mortality, Across Patient Demographics, and Hospital Ownership

\begin{tabular}{|c|c|c|c|c|c|c|c|}
\hline & \multirow[t]{2}{*}{ Private } & \multicolumn{2}{|l|}{ Medicare } & \multicolumn{2}{|l|}{ Medicaid } & \multicolumn{2}{|l|}{ Uninsured } \\
\hline & & OR $(95 \% \mathrm{CI}) *$ & $p$ & OR $(95 \% \mathrm{CI})^{*}$ & $p$ & OR $(95 \% \mathrm{CI}) *$ & $p$ \\
\hline Age $<35$ & 1.0 (reference) & $1.17(0.85-1.61)$ & 0.326 & $1.13(0.95-1.35)$ & 0.167 & $1.28(0.98-1.70)$ & 0.08 \\
\hline Age $35-55$ & 1.0 (reference) & $1.15(1.02-1.29)$ & 0.021 & $1.17(1.04-1.31)$ & 0.009 & $1.27(1.09-1.48)$ & 0.002 \\
\hline Age $>55$ & 1.0 (reference) & $1.05(0.99-1.12)$ & 0.058 & $1.08(0.97-1.19)$ & 0.152 & $1.33(1.16-1.52)$ & $<0.001$ \\
\hline \multicolumn{8}{|l|}{ Gender } \\
\hline Male & 1.0 (reference) & $1.06(0.99-1.13)$ & 0.06 & $1.15(1.06-1.27)$ & 0.002 & $1.19(1.06-1.34)$ & 0.003 \\
\hline Female & 1.0 (reference) & $1.08(1.01-1.17)$ & 0.026 & $1.06(0.98-1.18)$ & 0.197 & $1.37(1.18-1.59)$ & $<0.001$ \\
\hline \multicolumn{8}{|l|}{ Race } \\
\hline White & 1.0 (reference) & $1.08(1.01-1.17)$ & 0.026 & $1.12(1.02-1.22)$ & 0.014 & $1.2(1.07-1.34)$ & 0.002 \\
\hline Black & 1.0 (reference) & $0.96(9.83-1.12)$ & 0.651 & $1.14(0.95-1.35)$ & 0.14 & $1.28(1.0-1.65)$ & 0.05 \\
\hline Hispanic & 1.0 (reference) & $1.13(0.95-1.35)$ & 0.14 & $1.09(0.98-1.33)$ & 0.409 & $1.27(0.95-1.70)$ & 0.12 \\
\hline Other & 1.0 (reference) & $1.07(9.89-1.27)$ & 0.47 & $1.03(0.84-1.27)$ & 0.29 & $1.54(1.16-2.04)$ & 0.003 \\
\hline \multicolumn{8}{|c|}{ Income quartile by home zip code } \\
\hline Quartile 1 & 1.0 (reference) & $1.06(0.96-1.18)$ & 0.224 & $1.12(0.98-1.28)$ & 0.088 & $1.21(1.01-1.45)$ & 0.034 \\
\hline Quartile 2 & 1.0 (reference) & $1.01(0.93-1.11)$ & 0.33 & $1.06(0.94-1.19)$ & 0.373 & $1.19(1.01-1.40)$ & 0.038 \\
\hline Quartile 3 & 1.0 (reference) & $1.13(1.03-1.25)$ & 0.013 & $1.13(0.97-1.31)$ & 0.095 & $1.26(1.04-1.52)$ & 0.02 \\
\hline \multirow{2}{*}{\multicolumn{8}{|c|}{ Referring hospital location }} \\
\hline & & & & & & & \\
\hline ED & 1.0 (reference) & $1.07(0.98-1.17)$ & 0.06 & $1.21(1.09-1.35)$ & $<0.001$ & $1.41(1.25-1.59)$ & $<0.001$ \\
\hline Inpatient & 1.0 (reference) & $1.06(0.99-1.13)$ & 0.054 & $1.05(0.97-1.15)$ & 0.202 & $1.03(0.788-1.20)$ & 0.33 \\
\hline \multicolumn{8}{|c|}{ Referring hospital ownership } \\
\hline Investor owned & 1.0 (reference) & $1.14(1.03-1.26)$ & 0.014 & $1.15(0.98-1.34)$ & 0.014 & $1.14(0.94-1.39)$ & 0.198 \\
\hline Non profit & 1.0 (reference) & $1.03(0.97-110)$ & 0.259 & $1.10(1.01-1.20)$ & 0.025 & $1.27(1.13-1.44)$ & $<0.001$ \\
\hline Government & 1.0 (reference) & $1.22(1.05-1.41)$ & 0.009 & $1.08(0.90-1.30)$ & 0.372 & $1.44(1.14-1.83)$ & 0.002 \\
\hline \multicolumn{8}{|c|}{ Receiving hospital ownership } \\
\hline Investor & 1.0 (reference) & $1.21(1.08-1.35)$ & 0.001 & $1.25(1.01-1.52)$ & 0.037 & $1.11(0.84-1.45)$ & 0.455 \\
\hline Non profit & 1.0 (reference) & $1.04(0.98-1.11)$ & 0.148 & $1.09(1.00-1.18)$ & 0.045 & $1.27(1.13-1.43)$ & $<0.001$ \\
\hline Government & 1.0 (reference) & $1.05(0.93-1.18)$ & 0.423 & $1.01(0.87-1.17)$ & 0.855 & $1.26(1.04-1.51)$ & 0.015 \\
\hline
\end{tabular}

*OR and $p$ value by multi-level logistic regression adjusting for age, gender, race, hospital ownership, whether the patient was transferred from an emergency department, and Elixhauser comorbidities 
of higher mortality echo those illustrated 30 years ago. ${ }^{13}$ Transfers of particularly high-risk, high-complexity patients requiring uncompensated care may continue to unduly burden a municipal hospital system which serves as a large safety net for uninsured patients. ${ }^{26-29}$ Taken together, we conclude that EMTALA is insufficient to protect uninsured patients who require transfer between hospitals.

There are many explanations for this finding. First, the lack of preventative services may result in uninsured patients presenting late in the course of disease, warranting emergency transfer, and ultimately higher mortality despite the best efforts of referring hospital. Alternatively, the burden of uncompensated care may motivate earlier transfer, while diagnosis and stabilization are ongoing resulting in higher risk. In either case, lack of insurance coverage contributes to the occurrence of a high-risk transition of care where miscommunication is common and treatment delay may result in worse outcomes.

In the current political climate, where insurance coverage expansion under the ACA is actively debated, it is important to understand the added risk associated with lack of insurance. While EMTALA mandates uncompensated care to patients who lack the ability to pay, it fails to systematize care in a way that mitigates the risk associated with an emergent interhospital transfer. Moreover, the burden of high-risk transfers falls disproportionately on government-financed hospitals, wherein emergency care and subsequent hospitalization and transfer are mandated by law. The lack of care coordination such as that occurs during inter-hospital transfers may contribute to less efficient care and worse outcomes for the uninsured.

This study has several limitations. As this is an observational study that relies on a single hospital, and validated in administrative data, there is no ability to assess the appropriateness of an individual patient transfer. Hospital transfer behavior is highly heterogeneous at the hospital level; diagnoses frequently change before and after transfer, and procedural rates are inconsistent. Elucidating the rationale for transfer among uninsured population is not possible in this study. Moreover, risk stratification via APACHE-II and Elixhauser comorbidities partly relies on prior diagnosis of chronic diseases, which may not be identified in the uninsured population that lack prior care, thus potentially biasing our conclusions. Additionally, the higher rate of mortality and early transfer may represent the best efforts of a hospital to transfer the patient to a higher level of care. In this case, adding additional barriers to transfer such as financial clearance may worsen disparities in the uninsured.

This study suggests reducing uninsured rate may reduce the need for high-risk inter-hospital transfers; however due to the observational design, it cannot identify a causative relationship between insurance coverage and transfers. Investigating the absolute impact of the Medicaid expansion on hospital to hospital transfer rates and subsequent outcomes is an important and necessary next step. Additionally, investigating hospital ownership, changes in financial health, and hospital transfer patterns would shed further light on this underappreciated public health challenge.

\section{CONCLUSION}

Incomplete insurance coverage is an important contributor to economic health disparities in the USA. We demonstrate that uninsured patients are frequently transferred prior to admission and earlier in their hospital stay and have worse outcomes despite EMTALA. Further expanding insurance coverage will allow for better systematization of already federally mandated inpatient care, likely reduce the rate of unnecessary transfers, thereby improving overall inpatient outcomes, and unburdening the municipal hospital system.

\section{Acknowledgments:}

We would like to thank Anne Marie Webber-Main $\mathrm{PhD}$ for her critical review of a draft of this paper and writing clarification.

Prior Presentation: This paper was presented at the Society of General Internal Medicine National Meeting in May 2016.

Corresponding Author: Michael G. Usher, MD PhD; Department of Medicine, Division of General Internal Medicine University of Minnesota Medical School, Minneapolis, MN, USA (e-mail: mgusher@umn. edu).

Funding Funding support for this study was provided by the NIH Clinical and Translational Science Award at the University of Minnesota: 8UL1TR000114-02.

\section{Compliance with Ethical Standards:}

Conflict of Interest: The authors declare that they have do not have a conflict of interest.

\section{REFERENCES}

1. Chen SI, Wang $\mathbf{Y}$, Dreyer $\mathbf{R}$, Strait KM, Spatz ES, Xu X, et al. Insurance and Prehospital Delay in Patients $</=55$ Years With Acute Myocardial Infarction. Am J Cardiol. 2015;116(12):1827-32.

2. Christopher AS, McCormick D, Woolhandler S, Himmelstein DU, Bor DH, Wilper AP. Access to Care and Chronic Disease Outcomes Among Medicaid-Insured Persons Versus the Uninsured. Am J Public Health. 2016;106(1):63-9.

3. Kangovi S, Barg FK, Carter T, Levy $K$, Sellman J, Long JA, et al. Challenges faced by patients with low socioeconomic status during the post-hospital transition. J Gen Intern Med. 2014;29(2):283-9.

4. Dombrovskiy VY, Martin AA, Sunderram J, Paz HL. Occurrence and outcomes of sepsis: influence of race. Crit Care Med. 2007;35(3):763-8.

5. Fowler RA, Noyahr LA, Thornton JD, Pinto R, Kahn JM, Adhikari NK, et al. An official American Thoracic Society systematic review: the association between health insurance status and access, care delivery, and outcomes for patients who are critically ill. Am J Respir Crit Care Med. 2010;181(9):1003-11.

6. Guadagnolo BA, Liao KP, Giordano SH, Elting LS, Shih YC. Variation in intensity and costs of care by payer and race for patients dying of cancer in Texas: an analysis of registry-linked medicaid, medicare, and dually eligible claims data. Med Care. 2015;53(7):591-8.

7. Kumar G, Taneja A, Majumdar T, Jacobs ER, Whittle J, Nanchal R, et al. The association of lacking insurance with outcomes of severe sepsis: retrospective analysis of an administrative database*. Crit Care Med. 2014;42(3):583-91.

8. Shah AA, Haider AH, Zogg CK, Schwartz DA, Haut ER, Zafar SN, et al. National estimates of predictors of outcomes for emergency general surgery. J Trauma Acute Care Surg. 2015;78(3):482-90; discussion 90-1.

9. Woolhandler S, Himmelstein DU. The Relationship of Health Insurance and Mortality: Is Lack of Insurance Deadly?, Ann Intern Med. 2017. 
10. Lochner P. Hill-Burton enforcement: a proposed remedy to cure hospital inertia. J Legis. 1987; 14(1):5.

11. Hyman DA, Studdert DM. Emergency Medical Treatment and Labor Act: what every physician should know about the federal antidumping law. Chest. 2015;147(6):1691-6.

12. Obama B. United States health care reform: progress to date and next steps. JAMA. 2016;316(5):525-32.

13. Schiff RL, Ansell DA, Schlosser JE, Idris AH, Morrison A, Whitman S. Transfers to a public hospital. A prospective study of 467 patients. N Engl J Med. 1986;314(9):552-7.

14. Cheng TC, Haisken-DeNew JP, Yong J. Cream skimming and hospital transfers in a mixed public-private system. Soc Sci Med. 2015;132:156-64.

15. Bayindir EE. Hospital ownership type and treatment choices. J Health Econ. 2012;31(2):359-70.

16. Cook SF, Visscher WA, Hobbs CL, Williams RL, Project ICIC. Project IMPACT: results from a pilot validity study of a new observational database. Crit Care Med. 2002;30(12):2765-70.

17. HCUP National Inpatient Sample (NIS). Agency for Healthcare Research and Quality, Rockville, MD. at: www.hcup-us.ahrq.gov/nisover-view.jsp.: Healthcare Cost and Utilizaltion Project (HCUP). 2012.

18. Usher MG, Fanning C, Wu D, Muglia C, Balonze K, Kim D, et al. Information handoff and outcomes of critically ill patients transferred between hospitals. J Crit Care. 2016;36:240-5.

19. Sokol-Hessner L, White AA, Davis KF, Herzig SJ, Hohmann SF. Interhospital transfer patients discharged by academic hospitalists and general internists: Characteristics and outcomes. J Hosp Med. 2016;11(4):245-50.

20. Hanmer J, Lu X, Rosenthal GE, Cram P. Insurance status and the transfer of hospitalized patients: an observational study. Ann Intern Med. 2014;160(2):81-90.
21. Kindermann DR, Mutter RL, Cartwright-Smith L, Rosenbaum S, Pines JM. Admit or transfer? The role of insurance in high-transfer-rate medical conditions in the emergency department. Ann Emerg Med. 2014;63(5):561-71 e8.

22. Kindermann DR, Mutter RL, Houchens RL, Barrett ML, Pines JM. Emergency department transfers and transfer relationships in United States hospitals. Acad Emerg Med. 2015;22(2):157-65.

23. Spain DA, Bellino M, Kopelman A, Chang J, Park J, Gregg DL, et al. Requests for 692 transfers to an academic level I trauma center: implications of the emergency medical treatment and active labor act. J Trauma. 2007;62(1):63-7; discussion 7-8.

24. HCUP State Inpatient Satabase (SID) and State Emergency Department Database (SEDD). Agency for Healthcare Research and Quality, Rockville, MD. at: www.hcup-us.ahrq.gov/nisover-view.jsp.: Healthcare Cost and Utilizaltion Project (HCUP). 2011-2013.

25. Knaus WA, Draper EA, Wagner DP, Zimmerman JE. APACHE II: a severity of disease classification system. Crit Care Med. 1985;13(10):818-29.

26. Encinosa WE, Bernard DM. Hospital finances and patient safety outcomes. Inquiry. 2005;42(1):60-72.

27. Kao DP, Martin MH, Das AK, Ruoss SJ. Consequences of federal patient transfer regulations: effect of the 2003 EMTALA revision on a tertiary referral center and evidence of possible misuse. Arch Intern Med. 2012;172(11):891-2.

28. Dong GN. Performing well in financial management and quality of care: evidence from hospital process measures for treatment of cardiovascular disease. BMC Health Serv Res. 2015;15:45.

29. Waters TM, Daniels MJ, Bazzoli GJ, Perencevich E, Dunton N, Staggs VS, et al. Effect of Medicare's nonpayment for Hospital-Acquired Conditions: lessons for future policy. JAMA Intern Med. 2015; 175(3):347-54. 